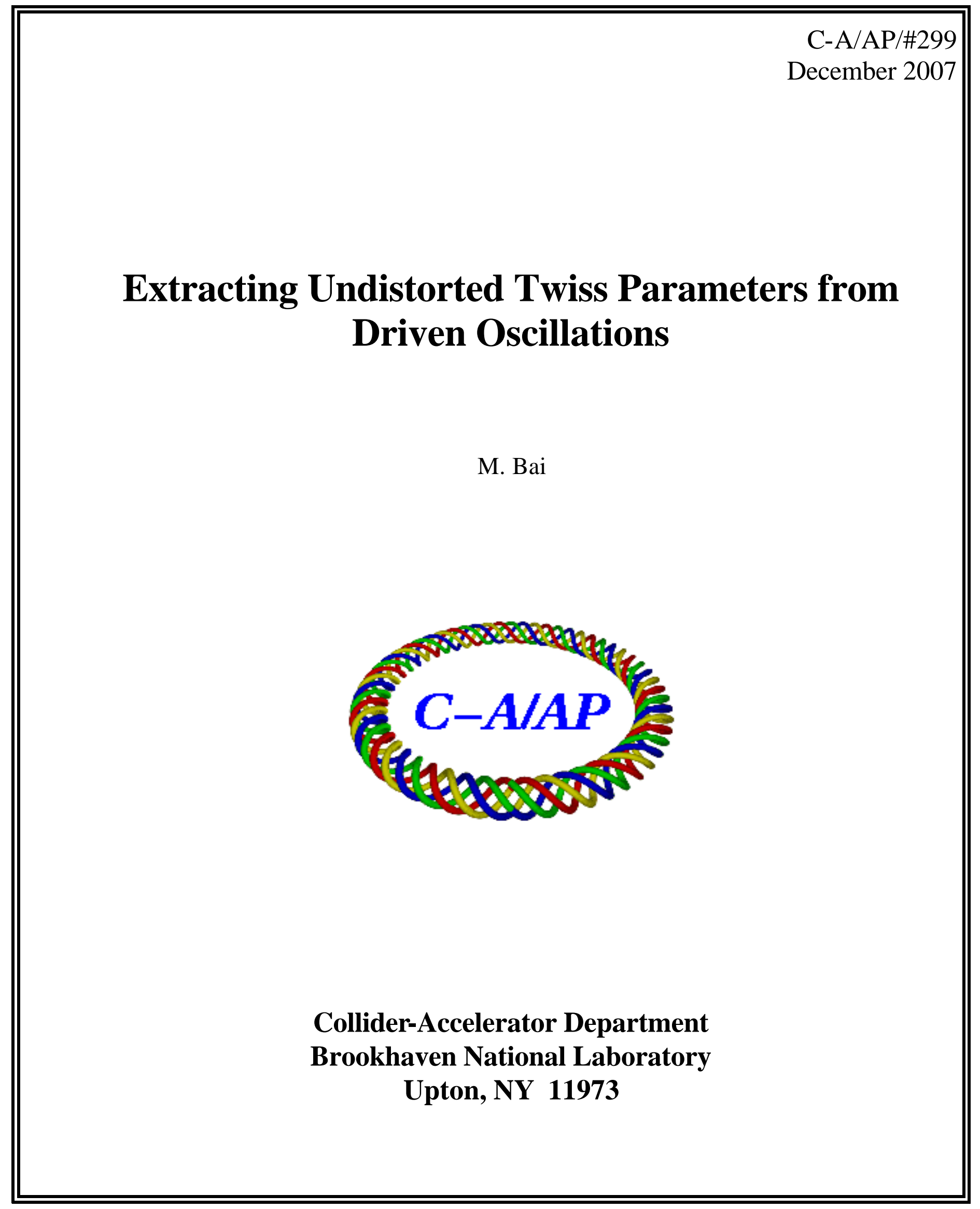




\title{
Extracting Undistorted Twiss Parameters from Driven Oscillations*
}

\author{
M. Bai \\ Brookhaven National Laboratory, Upton, NY 11973, U.S.A
}

\begin{abstract}
The technique of using an ac dipole driven coherent betatron oscillation to measure beta functions, as well as phase phase advances, has been applied in RHIC as well as in the Tevatron. This paper presents the difference between the optics measurement using a free betatron oscillation and using a driven coherent betatron oscillation. Techniques on how to extract undistorted beta functions and phase advances from the driven coherent betatron oscillation are discussed.
\end{abstract}

\section{INTRODUCTION}

The technique of using an ac dipole with an oscillating magnetic field to excite a coherent betatron oscillation was first tested and applied in the Brookhaven AGS [1]. Since then, it has been adopted by RHIC and by the Tevatron and will also be installed in the LHC [2-5]. A sizable long lasting coherence can be induced without blowing up the beam emittance when the driven coherence is excited adiabatically. This makes it more attractive for hadron accelerators to conduct optics measurements, and beam dynamics studies.

Without losing generality, assume a single ac dipole with vertical magnetic field in a linear accelerator. At every orbital revolution when a particle passes by the ac dipole, it gets an horizontal deflection $\Delta x_{p}^{a c}(k)$ given by

$$
\Delta x_{p}^{a c}(k)=\frac{B_{y}^{a c} L}{B \rho} \beta_{x}^{a c} \cos \left(2 \pi Q_{x}^{a c} k+\chi_{x}^{a c}\right)=\frac{B_{y}^{a c} L}{2 B \rho} \beta_{x}^{a c}\left(e^{i\left(2 \pi Q_{x}^{a c} k+\chi_{x}^{a c}\right)}+e^{-i\left(2 \pi Q_{x}^{a c} k+\chi_{x}^{a c}\right)}\right)
$$

where $k$ is the turn number, $B \rho$ is the magnetic rigidity, $\beta_{x}^{a c}$ is the horizontal beta function at the location of the ac dipole, $B_{y}^{a c} L$ is the amplitude of the ac dipole integrated oscillating field strength, $Q_{x}^{a c}$ is the ac dipole driving tune (the ac dipole field oscillating frequency divided by the revolution frequency) and $\chi_{x}^{a c}$ is the ac dipole oscillating field initial phase. Let

$$
z_{x}^{a c}=x^{a c}+i x_{p}^{a c}
$$

Here, $x^{a c}$ and $x_{p}^{a c}$ are the normalized coordinates at the location of the ac dipole. Since the transfer map from the exit of the ac dipole back to the entrance of the ac dipole in one turn is $e^{i 2 \pi Q_{x}}$, the normalized coordinates between a two successive turns are related by

$$
z_{x}^{a c}(k+1)=e^{i 2 \pi Q_{x} k}\left(z_{x}^{a c}(k)+\Delta x_{p}^{a c}(k)\right) .
$$

Here, $Q_{x}$ is the beam horizontal betatron tune. The solution of Eq. 3 in the condition of adiabatically ramping the ac dipole oscillating field from zero to $B_{y}^{a c} L$ yields the exact solution of the driven betatron oscillation as shown in Eq. 4.

$$
z_{x}^{a c}(k)=A_{m}^{a c} e^{i\left(2 \pi Q_{x}^{a c} k-\pi\left(Q_{x}^{a c}-Q_{x}\right)+\chi_{x}^{a c}\right)}-A_{p}^{a c} e^{-i\left(2 \pi Q_{x}^{a c} k-\pi\left(Q_{x}^{a c}+Q_{x}\right)+\chi_{x}^{a c}\right)} .
$$

with

$$
A_{m}^{a c}=\frac{B_{y}^{a c} L}{4 \sin \left(\pi\left(Q_{x}^{a c}-Q_{x}\right)\right) B \rho} \beta_{x}^{a c}
$$

and

$$
A_{p}^{a c}=\frac{B_{y}^{a c} L}{4 \sin \left(\pi\left(Q_{x}^{a c}+Q_{x}\right)\right) B \rho} \beta_{x}^{a c}
$$

\footnotetext{
${ }^{*}$ The work was performed under the auspices of the US Department of Energy.
} 
Here, one also assumes that the initial betatron oscillation is negligible. Detailed derivation can be found in the $\mathrm{RHIC} / \mathrm{AP} / 159$ [6]. The normalized coordinates $x^{a c}(k)$ and $x_{p}^{a c}(k)$ at the ac dipole are then

$$
\begin{aligned}
x^{a c}(k)= & \frac{B_{y}^{a c} L}{4 \sin \left(\pi\left(\mathrm{Q}_{\mathrm{x}}^{\mathrm{ac}}-\mathrm{Q}_{\mathrm{x}}\right)\right) \mathrm{B} \rho} \beta_{x}^{a c} \cos \left(2 \pi Q_{x}^{a c} k-\pi\left(Q_{x}^{a c}-Q_{x}\right)+\chi_{x}^{a c}\right)- \\
& \frac{B_{y}^{a c} L}{4 \sin \left(\pi\left(\mathrm{Q}_{\mathrm{x}}^{\mathrm{ac}}+\mathrm{Q}_{\mathrm{x}}\right)\right) \mathrm{B} \rho} \beta_{x}^{a c} \cos \left(2 \pi Q_{x}^{a c} k-\pi\left(Q_{x}^{a c}+Q_{x}\right)+\chi_{x}^{a c}\right) .
\end{aligned}
$$

and

$$
\begin{array}{r}
x_{p}^{a c}(k)=\frac{B_{y}^{a c} L}{4 \sin \left(\pi\left(Q_{x}^{a c}-Q_{x}\right)\right) B \rho} \beta_{x}^{a c} \sin \left(2 \pi Q_{x}^{a c} k-\pi\left(Q_{x}^{a c}-Q_{x}\right)+\chi_{x}^{a c}\right)+ \\
\frac{B_{y}^{a c} L}{4 \sin \left(\pi\left(Q_{x}^{a c}+Q_{x}\right)\right) B \rho} \beta_{x}^{a c} \sin \left(2 \pi Q_{x}^{a c} k-\pi\left(Q_{x}^{a c}+Q_{x}\right)+\chi_{x}^{a c}\right) .
\end{array}
$$

For any other location in the machine, the normalized betatron coordinates are obtained through the rotation matrix between the ac dipole and the observation location $s$

$$
\left(\begin{array}{l}
x^{s} \\
x_{p}^{s}
\end{array}\right)=\sqrt{\frac{\beta_{x}^{s}}{\beta_{x}^{a c}}}\left(\begin{array}{cc}
\cos \Delta \psi_{s} & \sin \Delta \psi_{s} \\
-\sin \Delta \psi_{s} & \cos \Delta \psi_{s}
\end{array}\right)\left(\begin{array}{l}
x^{a c} \\
x_{p}^{a c}
\end{array}\right)
$$

which yields

$$
x^{s}(k)=A_{m} \cos \left(2 \pi Q_{x}^{a c} k-\pi\left(Q_{x}^{a c}-Q_{x}\right)+\chi_{x}^{a c}-\Delta \psi_{s}\right)-A_{p} \cos \left(2 \pi Q_{x}^{a c} k-\pi\left(Q_{x}^{a c}+Q_{x}\right)+\chi_{x}^{a c}+\Delta \psi_{s}\right)
$$

with

$$
A_{m}=\frac{B_{y}^{a c} L}{4 \sin \left(\pi\left(Q_{x}^{a c}-Q_{x}\right)\right) B \rho} \sqrt{\beta_{x}^{a c} \beta_{x}^{s}}
$$

and

$$
A_{p}=\frac{B_{y}^{a c} L}{4 \sin \left(\pi\left(Q_{x}^{a c}+Q_{x}\right)\right) B \rho} \sqrt{\beta_{x}^{a c} \beta_{x}^{s}} .
$$

Here, $\beta_{x}^{s}$ is the beta function at location $s . \Delta \psi_{s}$ is the phase advance between the ac dipole and location $s$. Evidently, the driven oscillation consists of two terms which have the same driving tunes but with different phases. Since in general one places the ac dipole driving tune very close to the betatron tune, the driven oscillation is dominated by the term with the amplitude $A_{m}$. However, the term with the amplitude $A_{p}$ can be non-negligible if the betatron tune is close to either an integer or a half integer.

\section{DERIVING THE COURANT-SNYDER TWISS PARAMETER FROM THE DRIVEN BETATRON OSCILLATION}

Since the amplitude of a free betatron oscillation is proportional to the square root of the beta function

$$
x^{s}(k)=\sqrt{2 \beta_{x}^{s} J_{x}} \cos \left(2 \pi Q_{x} k+\psi_{x}^{s}\right)
$$

one can then determine the ratio of the beta functions and the phase advance between any two locations of the machine. Here, $J_{x}$ is the action of the particle and $\psi_{x}^{s}$ is the phase advance at location $s$. Comparing the free oscillation in Eq. 13 with the driven oscillation in Eq. 10, these assumptions hold for each individual oscillation term with the amplitude of $A_{m}$ or $A_{p}$, respectively.

However, since both the primary term and the secondary term have the same frequency, Eq. 10 can also be expressed as a single oscillation term

$$
x^{s}(k)=A_{e f f} \cos \left(2 \pi Q_{x}^{a c} k+\psi_{e f f}^{s}\right) .
$$

In this case, the ratio $A_{\text {eff }}$ between two bpms is an approximation of the beta function ratio. And the phase difference between the two bpms is also an approximation of the betatron phase advance between them. The deviation of the 

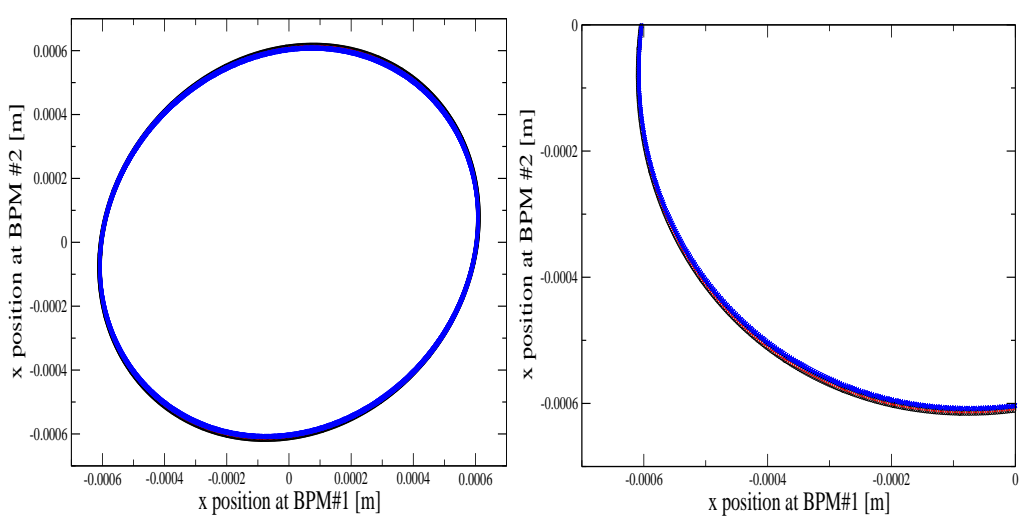

FIG. 1: The left plot shows the phase relation between two bpms by displaying the turn by turn beam position at bpm $\sharp 1$ and bpm $\sharp 2$. The right plot is the zoom of the lower left corner of the left plot. The data set in blue cross is obtained from a free betatron oscillation. The red diamonds represents the dominant oscillation term in Eq. 10 by fitting the turn by turn driven coherent oscillation with the exact initial term $\chi_{x}^{a c}$ in Eq. 16. The data set in black dot shows the fitting results of the dominant oscillation term in Eq. 10 but with $\chi_{x}^{a c}=0$ in Eq. 16. The difference between the three cases is negligible.

artificial beta function and phase advance is highly correlated with the betatron tune [7] and can be quite significant if the betatron tune is close to either an integer or a half integer.

There are at least two ways to extract the real beta function and phase advance from a driven betatron oscillation. Experimentally, one can take two measurements, one with the driving tune above the betatron tune and one with the driving tune below. The average of the two optics measurements removes this artificial beta beat if the machine is linear.

The difference can also be removed by fitting the turn-by-turn driven betatron oscillation with two specific oscillation terms,

$$
x_{s}(k)=A_{m}\left(\cos \left(2 \pi Q_{x}^{a c} k+\psi_{m}\right)-A_{p} \cos \left(2 \pi Q_{x}^{a c} k-2 \pi Q_{x}^{a c}+2 \chi_{x}^{a c}-\psi_{m}\right)\right)
$$

where $\psi_{m}=-\pi\left(Q_{x}^{a c}-Q_{x}\right)+\chi_{x}^{a c}-\Delta \psi_{s}$. Here, we assume that we know the ac dipole driving tune and the initial phase since the ac dipole is well controlled. This way, one obtains the amplitude and phase of the two oscillation terms in Eq. 15 by fitting the turn by turn driven betatron oscillation with Eq. 16

$$
\begin{array}{r}
x_{s}(k)=A_{m}\left\{\cos \left(\psi_{m}\right)\left[\cos \left(2 \pi Q_{x}^{a c} k\right)-r \cos \left(2 \pi Q_{x}^{a c} i-2 \pi Q_{x}^{a c}+2 \chi_{x}^{a c}\right)\right]-\right. \\
\left.\sin \left(\psi_{m}\right)\left[\sin \left(2 \pi Q_{x}^{a c} k\right)+r \sin \left(2 \pi Q_{x}^{a c} k-2 \pi Q_{x}^{a c}+2 \chi_{x}^{a c}\right)\right]\right\} .
\end{array}
$$

where

$$
r=\frac{\sin \left(\pi\left(Q_{x}^{a c}+Q_{x}\right)\right)}{\sin \left(\pi\left(Q_{x}^{a c}-Q_{x}\right)\right)}
$$

The amplitude and phase for the dominant term are then directly proportional to the beta function and the phase advance at the location $s$. Even though the accurate calculation of $\psi_{m}$ requires precise knowledge of the ac dipole initial phase $\chi_{x}^{a c}$, the relative phase advance between two locations is independent of $\chi_{x}^{a c}$. Fig. 1 shows the relative phase relation between the two beam position monitors (bpms) located at $s_{1}$ and $s_{2}$ for three different cases. It is evident that one can extract the phase advance between two bpms by fitting the driven betatron oscillation using $\chi_{x}^{a c}=0$ in Eq. 16.

Fig. 2 shows the calculated beta functions at each bpm and phase advances between each two consecutive bpms in the RHIC Blue ring. Results of using both Eq. 14 (black circles) and Eq. 16 (blue squares), are presented against the beta function and phase advance calculated from the RHIC model.

\section{CONCLUSION}

Because the ac dipole provides an oscillating deflection, the resulting driven coherent betatron oscillation contains two oscillation terms with the same frequency but different phases. This causes an artificial beta beat if the two terms are not separated. This effect can be experimentally removed by averaging two optics measurements with the ac dipole driving tune below and above the betatron tune. This paper shows that an alternative way is to fit the turn-by-turn beam position data with two oscillation terms. 


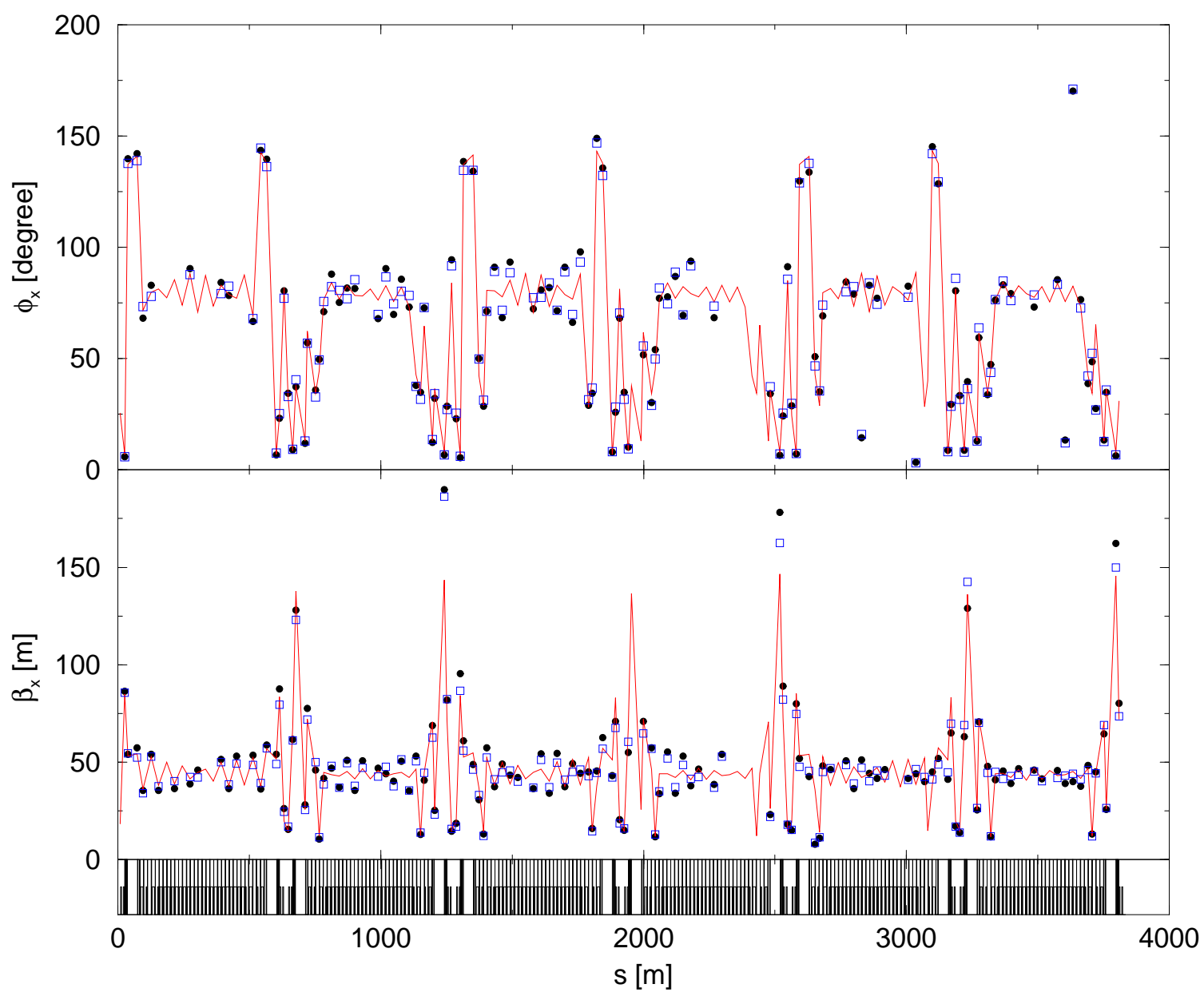

FIG. 2: The bottom plot shows the RHIC lattice as a function of the azimuthal coordinate $s$. The top plot is the calculated phase advance between two consecutive beam position monitors (bpm) calculated from the ac dipole driven coherent oscillation. The black dots correspond to fitting the ac dipole induced coherent oscillation with Eq. 14 and the open blue squares correspond to fitting the driven coherent oscillation with Eq. 16 assuming $\chi_{x}^{a c}=0.0$. The middle plot shows the measured beta functions at each BPM. Similar to the top plot, the black dots are the results using Eq. 14 and the blue squares show the results of fitting the coherent oscillation with Eq. 16 assuming $\chi_{x}^{a c}=0.0$.

\section{ACKNOWLEDGMENT}

The author would like to thank Dr. T. Roser and Dr. S. Peggs for the fruitful discussions. The author would also like to thank Dr. N. Abreu and Dr. G. Robert-Demolaize for proof reading.

[1] M. Bai et al Experimental test of coherence betatron excitations, Phys. Rev. E56, 6002, (1997)

[2] M. Bai et al RHIC AC Dipole Design and Construction, Proceedings of PAC01, 3606, Chicago, USA (2001)

[3] R. Miyamoto et al, Proceedings of PAC07, 3868, Albuquerque, New Mexico, USA (2007)

[4] O. Berrig et al Excitation of Large Transverse Beam Oscillations without Emittance Blow-up Using the AC-Dipole Principle, Proceedings of DIPAC 2001, 82, Grenoble, France (2001)

[5] J. -P. Koutchouk Advanced Diagnostics of Lattice Parameters in Hadron Colliders, Proceedings of DIPAC 2003, 45, Mainz, Germany (2003)

[6] S. Peggs and C. Tang Nonlinear Diagnostics Using an AC Dipole, RHIC/AP Report 159, (1998)

[7] R. Miyamoto et al, Proceedings of PAC07, 3465, Albuquerque, New Mexico, USA (2007) 